\title{
Análise da distribuição geográfica dos casos de hanseníase. Rio de Janeiro, 2001 a 2012
}

\author{
Analysis of the geographical distribution of cases of leprosy. \\ Rio de Janeiro, 2001-2012
}

Renata Gracie ${ }^{1}$

Julia Novaes de Barros Peixoto ${ }^{1}$

Fabiane Bertoni dos Reis Soares ${ }^{1}$

Mariana de Andrea Vilas-Boas Hacker ${ }^{2}$

${ }^{1}$ Instituto de Comunicação e Informação Científica e Tecnologia em Saúde, Fiocruz. Av. Brasil 4365, Manguinhos. 21040-360 Rio de Janeiro RJ Brasil. gracie.renata@gmail.com ${ }^{2}$ Instituto Oswaldo Cruz, Fiocruz. Rio de Janeiro RJ Brasil.

\begin{abstract}
Studies have demonstrated that the geographical distribution of leprosy is related to different socioeconomic factors. This article aims to study the geographical distribution of leprosy in the state of Rio de Janeiro. The cases of leprosy reported in the 2001-2012 period were mapped according to municipality. Epidemiological and socioeconomic indicators were calculated. The ArcMap program was used for the construction of maps and Earth View to calculate the Bayesian rate. It was observed that leprosy is presented in hyper-endemic levels especially in the metropolitan area. However, there is also a reduction of the detection rate in the most recent study period. In municipalities in the metropolitan region and the north western region detection in children under 15 is high, indicating an active transmission situation. In municipalities in the south-central region and especially in the coastal region, there was a high proportion of cases diagnosed with level II disability, reflecting late diagnosis. There was no linear correlation between socioeconomic indicators and leprosy rate. These results contribute to the analysis of the geographical distribution of leprosy, important for the identification of areas for resource allocation, aiming to control and eliminate the disease.
\end{abstract}

Key words Leprosy, Geoprocessing, Indicators
Resumo Trabalhos demonstraram que a distribuição geográfica da hanseníase está relacionada a diferentes fatores socioeconômicos. O objetivo deste artigo é estudar a distribuição geográfica da hanseníase no estado do Rio de Janeiro. Os casos de hanseníase notificados no período 2001-2012 foram mapeados segundo município. Foram calculados indicadores epidemiológicos e socioeconômicos. Utilizou-se o programa ArcMap para a construção dos mapas e o Terra View para o cálculo de taxa bayesiana. Observou-se que a hanseníase apresenta-se em níveis hiperendêmicos, especialmente na região metropolitana. No entanto, observa-se também uma redução do coeficiente de detecção no período mais recente do estudo. Em municípios da região metropolitana e da região noroeste a detecção em menores de 15 anos é elevada, indicando situação de transmissão ativa. Em municípios da região centro-sul e especialmente na baixada litorânea, observou-se elevada proporção de casos diagnosticados com grau II de incapacidade, refletindo alto índice de diagnóstico tardio. Não foi observada correlação linear entre os indicadores socioeconômicos e a detecção da hanseniase. Esses resultados contribuem para a análise da distribuição geográfica da hanseníase, importante para a identificação de áreas para alocação de recursos, visando controle e eliminação da doença.

Palavras-chave Hanseníase, Geoprocessamento, Indicadores 


\section{Introdução}

Sabe-se que a Hanseníase ainda constitui um relevante problema de saúde pública. O Brasil se mantem como segundo país com maior número de casos novos detectados no mundo, precedido pela Índia. Embora a detecção anual venha declinando, no início de 2010 a prevalência global de hanseníase era de cerca de 212 mil casos. No Brasil, em 2014, dos 31.064 casos novos detectados, 2.341 foram em menores de 15 anos e o coeficiente de detecção geral foi de 15,32 para cada 100 mil habitantes ${ }^{1,2}$.

Alguns municípios no Brasil já alcançaram a meta de eliminação da doença, no entanto, as regiões Norte, Centro-Oeste e Nordeste ainda apresentam altos patamares de casos da doença. Apesar da tendência de estabilização dos coeficientes de detecção no Brasil, os coeficientes apresentam grande variação nas diferentes regiões brasileiras $^{3}$.

Em 2014, no estado do Rio de Janeiro, o coeficiente de prevalência foi de 0,55 por 10.000 habitantes, em menores de 15 anos de 1,86 por 100.000 habitantes; e o de grau 2 de incapacidade de 0,75 para cada 100.000 habitantes ${ }^{1}$.

Fatores como densidade populacional, hábitos de vida, aspectos culturais, condições sanitárias e de moradia devem ser ponderados em um cenário desfavorável como o apresentado pelo estado do Rio de Janeiro".

Estudos demonstram que a hanseníase é fortemente relacionada com as condições de vida e pobreza $^{5,6}$. Segundo Cunha et al. ${ }^{4}$, evidencia-se uma tendência de concentração dos doentes em camadas da sociedade menos favorecidas.

Nos últimos anos a epidemiologia social, área da epidemiologia que estuda a distribuição social e os determinantes sociais de saúde, tem se tornado proeminente e incorporado novas ferramentas de análise ${ }^{7}$.

Apesar da epidemiologia moderna focalizar prioritariamente fatores de risco e comportamentos individuais, os determinantes fundamentais de saúde, ao nível populacional, estão relacionados a fatores sociais, econômicos e ambientais. O contexto social (demográfico, econômico e político) influencia a epidemiologia dos diferentes agravos à saúde e modula comportamentos individuais que afetam a saúde populacional ${ }^{8}$.

No entanto, o desenvolvimento de cada doença é um fenômeno biológico individual, é possível que para diversas doenças, se não para todas, estes determinantes não possam ser inteiramente operacionalizados apenas ao nível individual ${ }^{9}$. Em se tratando de doenças infecciosas a dimensão ecológica é indispensável, pois a determinação é, inevitavelmente, individual e coletiva ${ }^{10}$. As diversas doenças infecciosas com longo período de incubação apresentam diferente distribuição em função das características geográficas e econômicas ${ }^{11}$.

As diferenças municipais referentes a vários agravos de saúde sugerem que fatores inter e intraurbanos (por exemplo, segregação residencial) desempenham um importante papel na saúde coletiva, indicando que é a multiplicidade e a interação de fatores em diferentes níveis que determinam a saúde das populações urbanas ${ }^{12}$.

Alguns trabalhos internacionais e nacionais demonstraram a heterogeneidade da distribuição geográfica da hanseníase e os fatores relaciona$\operatorname{dos}^{5,6,13-30}$. A avaliação da distribuição geográfica permite identificar áreas com maior concentração de casos visando melhor alocação de recursos para o controle da doença.

O Ministério da Saúde recomenda o uso dos indicadores epidemiologicos para monitoramento e avaliação da endemia, sendo recentemente ressaltada a importância da avaliação do coeficiente de detecção em menores de 15 anos e proporção de casos diagnosticados com grau 2 de incapacidade ${ }^{1}$.

Os indicadores podem ser utilizados para ajudar a descrever uma determinada situação e para acompanhar mudanças ou tendências em um período de tempo. Os indicadores de saúde permitem a comparabilidade entre diferentes áreas ou diferentes momentos e fornecem subsídios ao planejamento das ações de saúde ${ }^{31}$.

O objetivo do presente estudo foi estudar a distribuição espacial da Hanseníase no estado do Rio de Janeiro através dos dados do Sistema Nacional de Agravos de Notificação-SINAN no período de 2001 a 2012.

\section{Metodologia}

Foi realizado um estudo ecológico em que os casos de hanseníase notificados no SINAN (Sistema Nacional de Agravos de Notificação), no período de 2001 a 2012, no estado do Rio de Janeiro, foram obtidos do site do DATASUS (www. datasus.gov.br). Os dados obtidos referem-se aos casos já confirmados. O período refere-se ao ano de diagnostico. Portanto, fazem parte do estudo os casos registrados no SINAM confirmados até a data de coleta das informações. 
Levando-se em consideração as diferenças de contingente populacional, o número de residentes em cada município no período estudado, também foi adquirido para o cálculo de taxas para os períodos de 2001 a 2006 e 2007 a 2012. Os dados referentes a populações municipais foram obtidos no site do Instituto Brasileiro de Geografia e Estatística (IBGE). Foram calculados os seguintes indicadores: coeficiente médio de deteç̧ão geral e em menores de 15 anos e proporção de casos com grau 2 de incapacidade nos dois períodos citados.

Para inserir os dados no mapa de municípios do estado do Rio de Janeiro utilizou-se o código do município como geocódigo. Com os dados calculados e georreferenciados a partir do código de município foram construídos os mapas utilizando um programa em ambiente de Sistemas de Informações Geográficas-SIG, ArcMap versão 10.

Utilizou-se também um outro programa em ambiente de SIG Terra View 4.2.0. Para o cálculo de taxa bayesiana consideramos os anos de 2004 e 2010, que correspondem ao meio dos dois períodos adotados.

O método bayesiano permite que seja feita uma comparação minimizando o impacto da variabilidade dos dados, especialmente presente em municípios de pequeno porte, cujo contingente populacional e número de casos pode ser muito reduzido, evitando imprecisão nas estimativas. As taxas corrigidas são menos instáveis, pois levam em conta no seu cálculo não só a informação da área, mas também a de sua vizinhança, incorporando dessa forma a correlação espacial ${ }^{32}$.

Foram acrescentadas as principais rodovias de forma a entender a distribuição, pois notamos uma concentração número de casos em municípios cortados por estas, partindo do pressuposto que as mesmas influenciam no acesso aos locais de saúde. Inserimos também a camada de grandes áreas verdes, que configuram uma barreira física natural entre os municípios.

Indicadores municipais foram calculados a fim de se verificar a correlação entre fatores socioeconômicos e o coeficiente de detecção da hanseníase. Foram analisados os seguintes indicadores municipais relacionados à situação socioeconômica: proporção de domicílios com responsável alfabetizado, proporção de domicílios com banheiro ou sanitário e esgotamento sanitário via rede geral de esgoto ou pluvial, média da renda mensal das pessoas responsáveis por domicílios e densidade populacional.

Os dados relativos aos fatores socioeconômicos foram obtidos por meio do site do IBGE e são referentes aos anos de realização dos censos. Os indicadores foram calculados a partir dos dados do censo de 2010 e foram correlacionados com os indicadores epidemiológicos nos dois períodos avaliados (2001-2006 e 2007-2012).

Utilizou-se o coeficiente de correlação linear de Spearman e considerou-se um nível de significância de $5 \%$. O coeficiente de correlação varia entre -1 e +1 e indica o grau de associação linear entre os indicadores e a detecção de hanseníase. Valores próximos à unidade indicam forte correlação, quando positivos esta é direta e quando negativo é indireta, isto é, inversamente proporcional.

O coeficiente de Spearman é menos sensível a valores extremos do que o coeficiente de Pearson, os dados em questão apresentam grande variabilidade de valores entre os municípios, devido à heterogeneidade da endemia e desigualdades socioeconômicas.

\section{Aspectos éticos}

Foram utilizados dados secundários disponíveis para consulta nos sites das instituições responsáveis pela coleta e divulgação dos mesmos. Os dados são obtidos de maneira agregada, não havendo qualquer acesso a informações pessoais dos casos e dos residentes dos municípios. Por se tratar de um estudo ecológico, nenhuma conclusão pode ser diretamente inferida ao nível individual.

\section{Resultados}

A Figura 1 apresenta o coeficiente bruto de detecção de hanseníase nos dois períodos de 2001 a 2006 e 2007 a 2012 e a distribuição da análise bayesiana nos anos de 2004 e 2010, que correspondem ao meio dos dois períodos adotados.

Observa-se que a região metropolitana concentra coeficientes elevados, em nível hiperendêmico (acima de 40/100 mil). As regiões norte e noroeste do estado também apresentam municípios com altos níveis de detecção de casos novos.

Observa-se também que no segundo período avaliado houve uma redução dos níveis dos coeficientes na maioria dos municípios.

Ao compararmos os coeficientes brutos com aqueles estimados pelo método bayesiano, não observamos diferença importantes em relação à detecção na população geral, portanto os dados brutos podem ser diretamente interpretados.

A Figura 2 apresenta a detecção de hanseníase em menores de quinze anos nos dois períodos de 

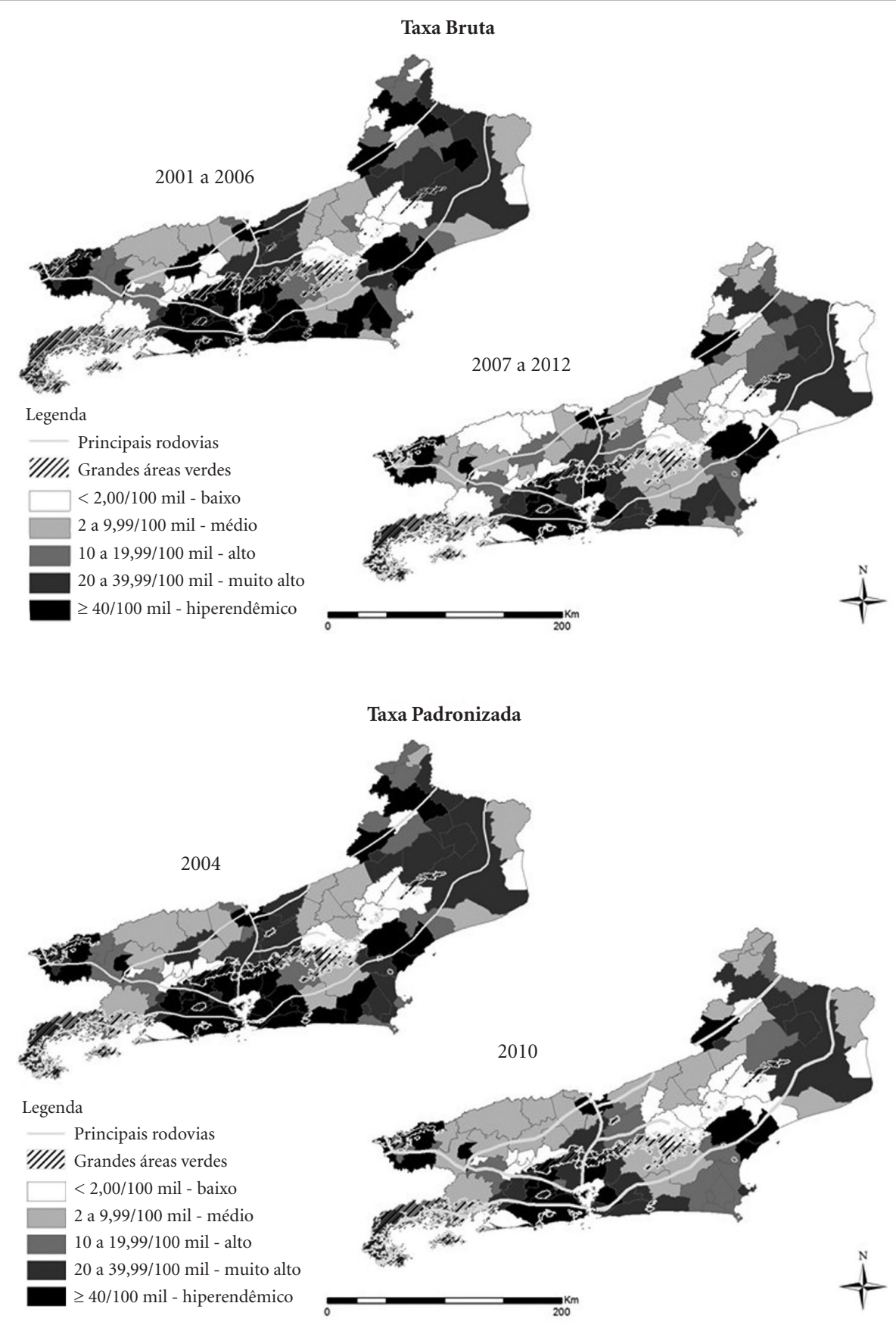

Figura 1. Coeficiente bruto e padronizado da detecção de hanseníase nos municípios do Rio de Janeiro, 2001 a 2012.

2001 a 2006 e 2007 a 2012 e a taxa ajustada pelo método bayesiano para os anos de 2004 e 2010.

Em relação à detecção em menores de 15 anos, observamos que a região noroeste do estado apresenta os níveis mais elevados de detecção nessa faixa etária, em níveis considerados alto e muito alto, especialmente no período de 2001 a 2006. A região metropolitana também apresentou alto nível de endemicidade para a detecção em menores de 15 anos, bem como alguns municípios da região noroeste. Entretanto, observa-se uma redução significativa na detecção entre os dois perío- 


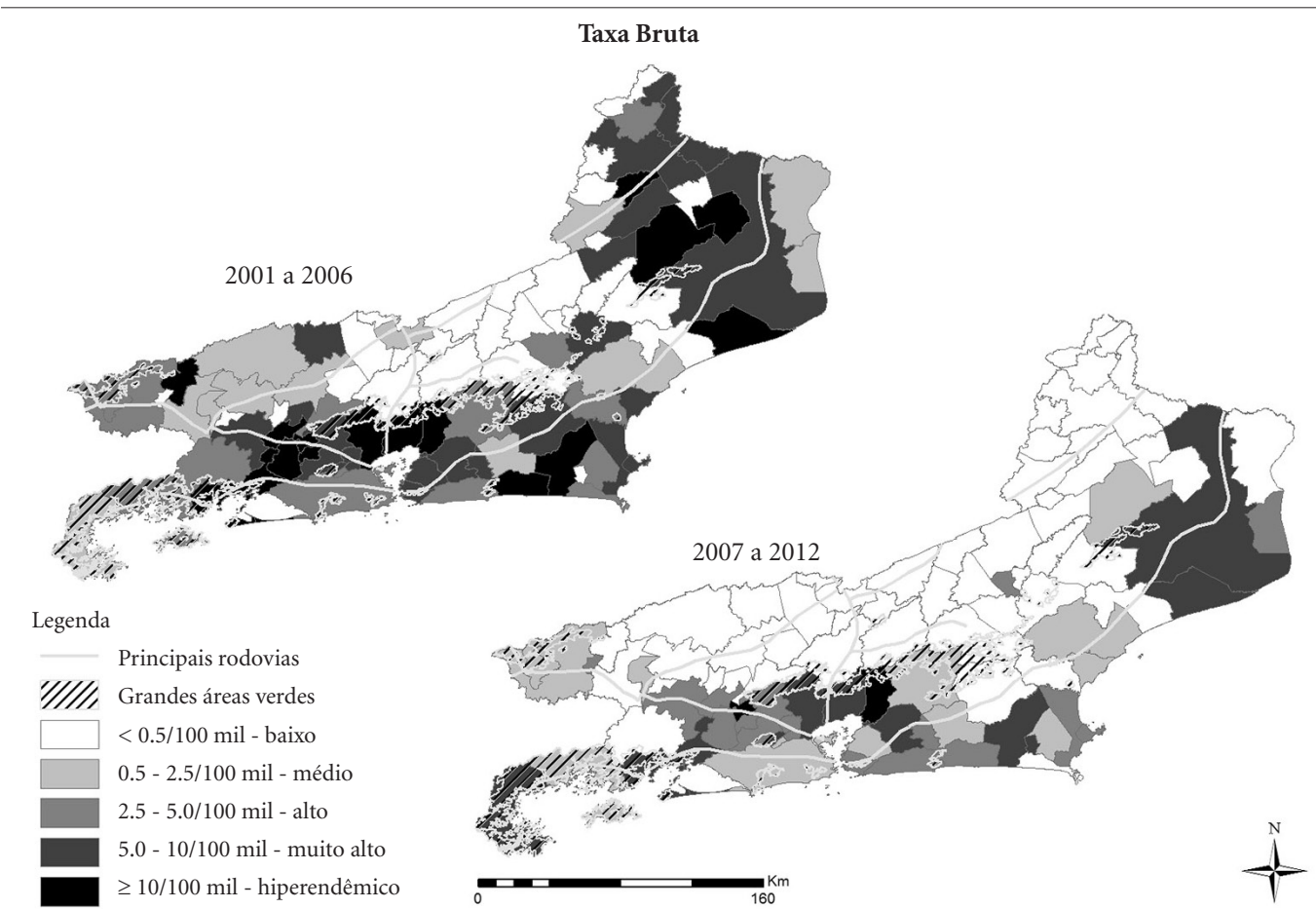

Taxa Padronizada

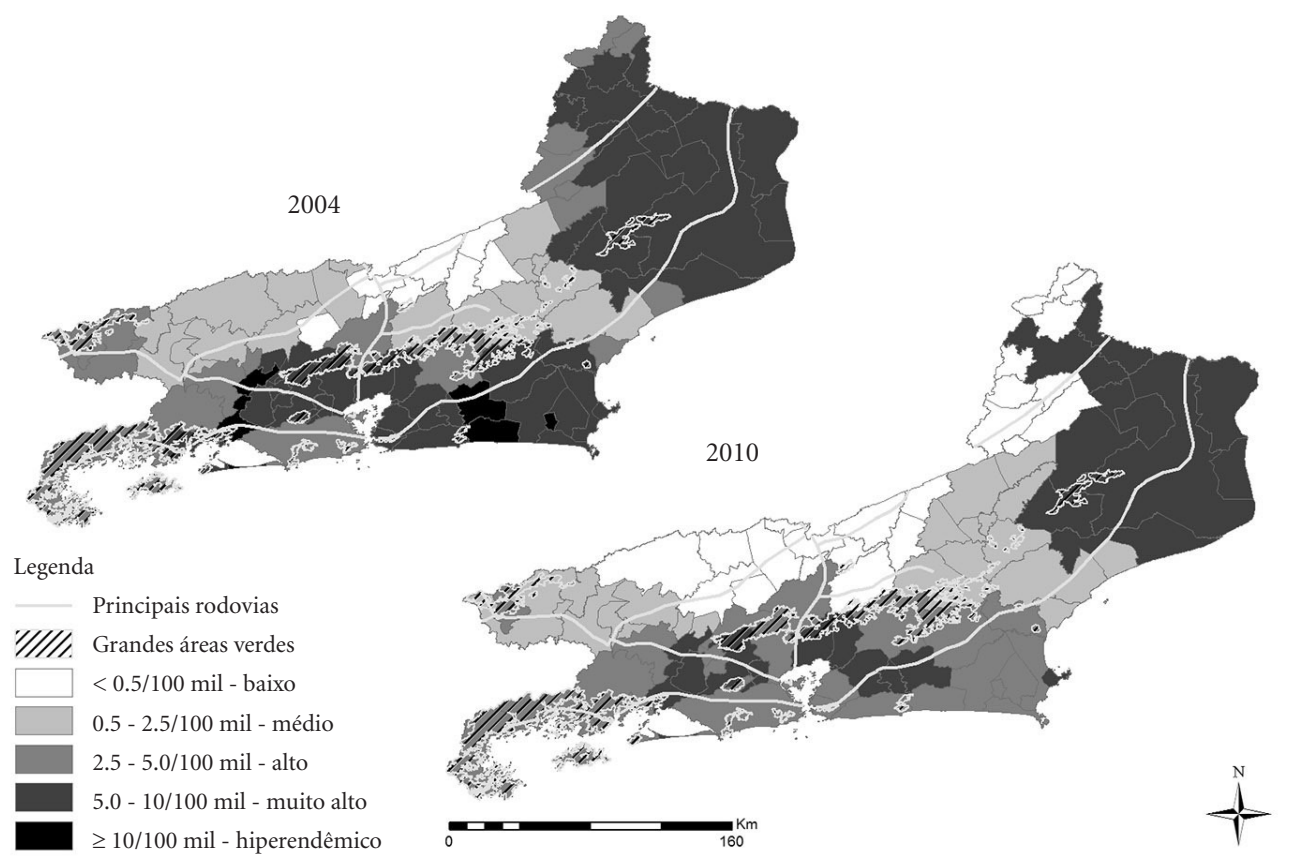

Figura 2. Coeficiente bruto e padronizado da detecção de hanseníase em menores de 15 anos. Rio de Janeiro, 2001 a 2012.

dos analisados em todo o estado. Ao analisarmos os coeficientes estimados pelo método bayesiano, essas diferenças observadas ficam mais evidentes.
Na Figura 3, observa-se que muitos municípios apresentam alta proporção de casos diagnosticados com grau II de incapacidade, ou seja, o diagnostico 
Proporção Grau de Incapacidade II

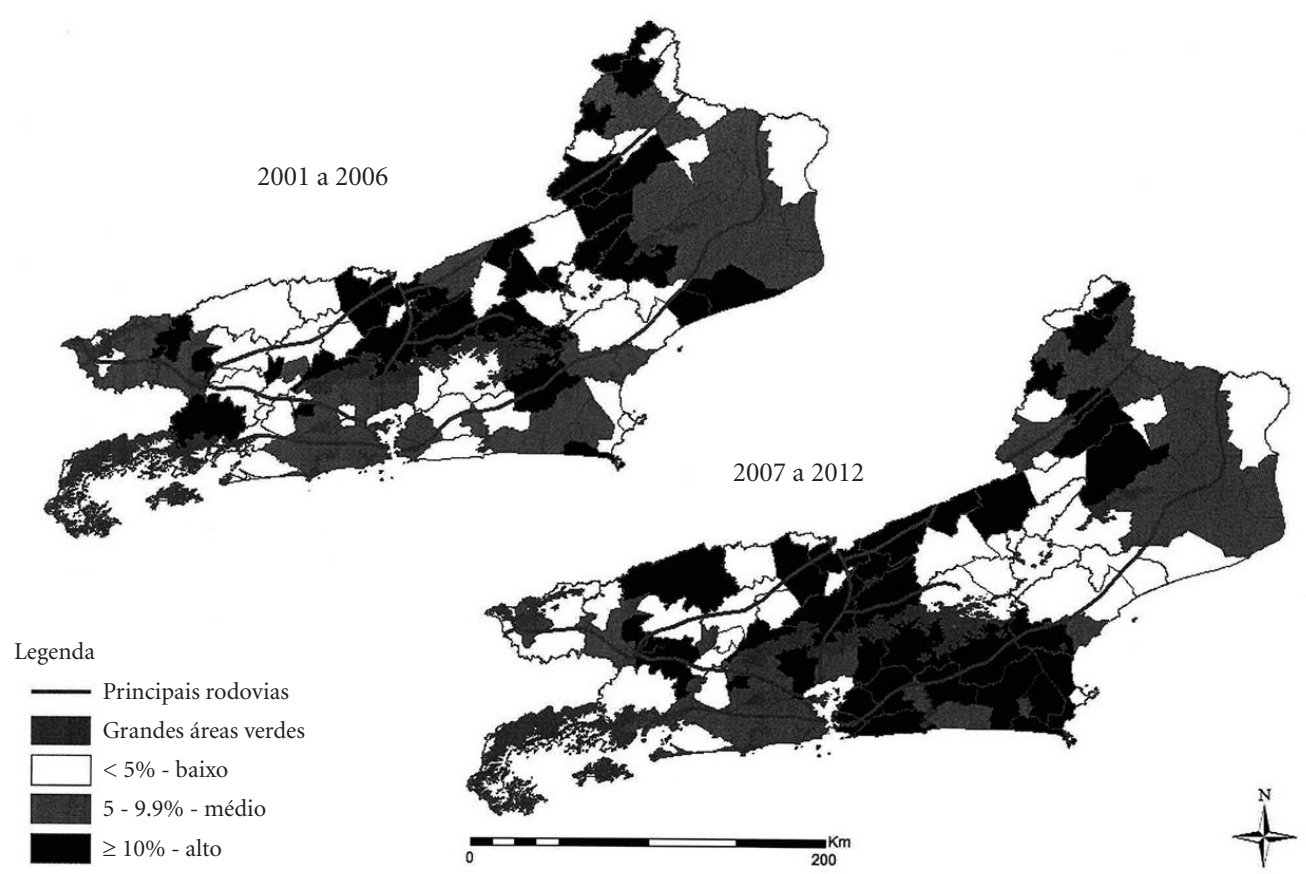

Figura 3. Proporção de casos diagnosticados com grau II de incapacidade. Rio de Janeiro, 2001 a 2012.

tardio vem se mantendo também no período mais recente, inclusive com aumento da proporção nas regiões centro-sul fluminense e das baixadas litorâneas. Considera-se que uma proporção de casos diagnosticados com grau II de incapacidade igual ou acima de $10 \%$ é considerada alta, entre 5 e 9,99, média, e menor do que $5 \%$ como baixa.

Na Tabela 1, observa-se correlação negativa significativa entre o coeficiente bruto de detecção para ambos os períodos com a média da renda, densidade populacional e proporção de domicílios com responsáveis alfabetizados, em que a detecção aumenta com a diminuição da renda e da densidade populacional e quanto menor for a proporção de domicílios com chefes de família alfabetizados. Porém os valores dos coeficientes foram inferiores a 0,4 , indicando fraca correlação.

A correlação entre o coeficiente bruto de detecção em < 15 anos para o período de 2001 a 2006 e proporção de domicílios com banheiro ou sanitário e esgotamento sanitário via rede geral de esgoto ou pluvial foi negativa significativa, em que a detecção aumenta com a diminuição de domicílios com banheiros e esgotamento, porém novamente o valor baixo do coeficiente $(\mathrm{r}=$ $-0,271$ ) indica fraca correlação.

Observa-se correlação positiva significativa entre o coeficiente bruto de detecção em $<15$ anos para o período de 2007 a 2012 e a densidade populacional $(\mathrm{r}=0,586)$ e a proporção de domicílios com chefe alfabetizado ( $r=0,495)$, em que a detecção nos municípios aumenta com o aumento da densidade de população e com o aumento da renda.

Não houve correlação significativa entre as taxas ajustadas e os indicadores socioeconômicos. Possivelmente o ajuste dos coeficientes pelo método bayesiano suavizou as correlações observadas entre os diferentes níveis dos indicadores epidemiológicos municipais.

\section{Discussão}

Os resultados do presente trabalho contribuem para a análise da distribuição geográfica da han- 
Tabela 1. Coeficiente de correlação entre indicadores socioeconômicos e indicadores epidemiológicos da hanseníase. Rio de Janeiro, 2001 a 2012.

\begin{tabular}{llcccc}
\hline & Renda & Esgotamento & $\begin{array}{c}\text { Densidade } \\
\text { Populacional }\end{array}$ & Alfabetização \\
\hline $\begin{array}{l}\text { Coeficiente de detecção geral } \\
\text { Cruto }\end{array}$ & $-0,295^{* *}$ & $-0,066$ & $-0,338^{* *}$ & $-0,389^{* *}$ \\
& Ajustado & 0,134 & $-0,021$ & 0,011 & 0,043 \\
Coeficiente de detecção em $<15$ anos & Bruto & $-0,082$ & $-0,271^{* *}$ & 0,140 & 0,097 \\
& Ajustado & $-0,188$ & 0,027 & $-0,060$ & $-0,154$ \\
2007-2012 & & & & & $-0,265^{*}$ \\
Coeficiente de detecção geral & Bruto & $-0,218^{*}$ & $-0,036$ & $-0,259^{*}$ & 0,069 \\
& Ajustado & 0,136 & $-0,034$ & 0,031 & $0,495^{* *}$ \\
Coeficiente de detecção & Bruto & 0,170 & $-0,022$ & $0,586^{* *}$ & $-0,151$ \\
em $<15$ anos & Ajustado & $-0,131$ & 0,115 & $-0,100$ & \\
\hline$*$
\end{tabular}

${ }^{*} \mathrm{p}<0,05,{ }^{* *} \mathrm{p}<0,01$.

seníase, sendo importante para a identificação de áreas com maior concentração de casos e para a alocação de recursos visando controle e eliminação da doença.

A proporção de casos diagnosticados com incapacidade permite estimar a efetividade das atividades para a detecção precoce e estimar a endemia oculta. O coeficiente de detecção permite determinar a tendência da endemia e medir a intensidade das atividades de detecção ${ }^{31}$. A detecção em menores de 15 anos revela ocorrência de transmissão recente da doença, indicando uma endemia ativa, ou seja, com ocorrência de novos $\operatorname{casos}^{33}$

A partir dos mapas gerados no presente estudo, observou-se que a hanseníase apresenta-se em níveis hiperendêmicos em diversos municípios do estado, especialmente na região metropolitana. No entanto, observa-se também uma redução do coeficiente de detecção no período mais recente do estudo. Esforços vêm sendo realizados para alcance da meta de eliminação, porém, campanhas devem ser direcionadas de acordo com o perfil epidemiológico dos diferentes municípios do estado.

Observou-se que em municípios da região metropolitana e da região noroeste a detecção em menores de 15 anos é elevada, indicando situação de transmissão ativa. Já em municípios da região centro-sul e especialmente na baixada litorânea, observou-se elevada proporção de casos diagnosticados com grau II de incapacidade, indicando alto índice de diagnóstico tardio.

A região noroeste do estado do Rio de Janeiro tem se destacado recentemente em função do seu crescimento econômico, porém, associado a isto, observa-se um crescimento desordenado da população com concentração em áreas de risco social.

Em um estudo espacial sobre crescimento urbano e hanseníase realizado na cidade de $\mathrm{Ma}$ naus, fatores como o rápido crescimento da cidade nas décadas de 70/80 alteraram suas dimensões espaciais o que teve repercussão na dinâmica das questões de saúde. Isto justifica o estudo do processo de urbanização e a distribuição da hanseníase partindo do pressuposto que a maior concentração de casos ocorre em locais com piores condições de vida ${ }^{6}$.

Segundo Opromolla et al. ${ }^{14}$ os movimentos migratórios, do interior de São Paulo para áreas desenvolvidas no centro do estado, associados à fatores da economia, influem na propagação da hanseníase, assim como para Imbiriba et al. ${ }^{6}$ na cidade de Manaus.

Entre os indicadores socioeconômicos municipais, a densidade populacional é um importante indicador relacionado a doenças transmissíveis. A correlação negativa significativa entre a detecção (na população geral e entre menores de 15 anos) e a densidade populacional encontrada no presente estudo, pode ser explicada, uma vez que a taxa de prevalência pode ser maior nos municípios menores com uma atenção básica precária e difícil acesso às principais rodovias.

Também observou-se correlação entre maior detecção na população menor de 15 anos e maior média de renda do chefe do domicilio, o que pode sugerir que a detecção de casos recentes é maior em municípios com melhores condições econômicas. Porém, no presente estudo, os valores encontrados para os coeficientes de correla- 
ção não indicaram forte correlação linear entre a detecção da hanseníase e os indicadores socioeconômicos analisados. Este fato pode indicar, que o acesso ao diagnóstico de hanseníase na população com indicadores socioeconômico pode não estar ocorrendo devido à dificuldade de entrada nos sistemas de saúde.

Estudo de Cury et al. ${ }^{29}$ não encontrou associação entre a incidência de hanseníase e a densidade demográfica, embora tenha identificado clusters de hanseníase e associação com baixos níveis socioeconômicos. Além disso, observou-se disparidade entre os clusters de casos e a localização de serviços de saúde.

Em Recife foi realizado um estudo com aplicação do modelo Bayesiano empírico de análise espacial considerando casos novos em menores de 15 anos, sendo levantadas hipóteses para os altos índices de casos multibacilares encontrados nesta população. A elevada densidade populacional e as baixas condições de vida da região expuseram desde cedo as crianças a altas cargas bacilares e sobrecarga do sistema imunológico, assim como deficiências nutricionais ${ }^{34}$.

Em um estudo ecológico sobre desigualdades e hanseníase no estado do Ceará, conclui-se que as desigualdades econômicas, a falta de escolaridade e a acessibilidade ao serviço de saúde são preditores de alta incidência de hanseníase, sendo, assim, que o grau de desigualdade econômica foi claramente ligado à incidência de hanseníase, o que significa que quanto mais heterogênea a distribuição da renda em um município, maior a probabilidade de que essa enfermidade seja um importante problema de saúde ${ }^{5}$.

A correlação observada no presente estudo entre a detecção da hanseníase e os indicadores de escolaridade e renda foi significativa, porém a associação encontrada foi de baixa magnitude.

No entanto, diversos estudos realizados em outras regiões do país, demonstraram com clareza a existência dessa associação. Em um estudo de análise espacial, no estado de São Paulo, o Índice de Responsabilidade (resultado da média entre escolaridade, renda e longevidade) apresentou correlação positiva com a detecção de hanseníase ${ }^{16}$.

No estudo de Imbiriba et al. ${ }^{6}$, que conjugou dados do IBGE e epidemiológicos, a baixa escolaridade dos moradores estava associada a altas taxas de detecção de hanseníase.

A heterogeneidade espacial da distribuição da hanseníase também foi demonstrada em outro estudo, no qual localidades com baixo nível socioeconômico apresentaram taxas de detecção da hanseníase mais elevadas, reforçando a teoria de que condição socioeconômica precária esta associada com a ocorrência da hanseníase ${ }^{28}$.

Segundo Rodrigues-Junior et al. ${ }^{16}$, as desigualdades sociais e econômicas e as consequentes precárias condições de vida, aliadas a altas densidades demográficas decorrentes da dinâmica social por questões econômicas favorecem a incidência de hanseníase, hipótese levantada em vários estudos ${ }^{6,14,26}$. Assim como a heterogeneidade na distribuição de renda municipal que influencia a acessibilidade à educação também contribui para o aumento do risco individual para a hanseníase ${ }^{5}$.

Outro estudo recente mostrou que taxas mais elevadas foram associadas a municípios de maior porte e com maiores taxas de alfabetização, mais urbanizados e com maior desigualdade social ${ }^{27}$.

Porém, os resultados desses estudos, assim como os resultados apresentados no presente artigo, se limitam ao respectivo nível geográfico analisado e não permitem que a inferência dos resultados seja estendida para o nível individual, para isso estudos multiníveis devem ser empregados ${ }^{9}$.

No presente estudo, observou que o ajuste dos coeficientes pelo método bayesiano suavizou as correlações observadas entre os diferentes níveis dos indicadores epidemiológicos municipais. Embora essa suavização fosse esperada, uma vez que o método bayesiano considera a correlação existente entre os municípios vizinhos, no entanto, as desigualdades entre os municípios, tanto do ponto de vista epidemiológico como econômico e social, poderia estar sendo subestimada dessa forma. Porém, ao analisar os dados brutos, ainda assim a correlação entre os indicadores não se mostrou fortemente associada, portanto, as desigualdades encontradas não parecem ser totalmente explicadas pelos indicadores selecionados.

Além disso, outra limitação dos resultados encontrados no presente estudo refere-se ao fato de que os indicadores socioeconômicos do IBGE só são disponíveis para os anos de realização de censo (a cada 10 anos). Considerando o período do estudo (2001 a 2012), estão disponíveis dados de 2000 e 2010, portanto, optou-se por utilizar os dados de 2010 para correlacionar com os indicadores epidemiológicos calculados para os períodos de 2001-2006 e 2007-2012. Essa defasagem, por um lado, pode levar à dificuldade de interpretação dos resultados, por outro, os indicadores de determinado ano podem representar a situação de um período anterior ao ano de referência.

A inclusão do mapeamento geográfico da morbidade por hanseníase em grandes municí- 
pios e regiões metropolitanas como uma prioridade da vigilância, prevenção e controle das doenças transmissíveis contribui para a identificação de áreas para alocação de recursos, visando o controle e a eliminação da doença ${ }^{35}$. O Sistema de Informação Geográfica tem sido utilizado para o monitoramento, a avaliação e a distribuição espacial da hanseníase. A visualização espacial colabora no planejamento de programas e processos de decisão.

\section{Colaboradores}

RSG Gracie realizou a análise geográfica, colaborou na redação do artigo e na discussão dos resultados. J Peixoto e FB Reis participaram do geoprocessamento dos dados. MAVB Hacker realizou a análise estatística dos dados, a redação e a revisão do artigo.

\section{Referências}

1. Brasil. Ministério da Saúde (MS). Portal da Saúde. Situação epidemiológica. Dados 2014. [acessado 2014 dez 10]. Disponível em: http://portalsaude.saude.gov. br/index.php/o-ministerio/principal/leia-mais-o-ministerio/705-secretaria-svs/vigilancia-de-a-a-z/hanseniase/11298-situacao-epidemiologica-dados

2. Organização Mundial de Saúde (OMS). Global leprosy situation, 2010. Wkly Epidemiol Rec 2010, 35(85):337348.

3. Brasil. Ministério da Saúde (MS). Coeficientes de prevalência e detecção em hanseníase Brasil, 2005 a 2014. [acessado 2015 out 20]. Disponível em: http://portalsaude.saude.gov.br/images/pdf/2015/outubro/29/Gr-fico-Coef.\%20Preval\%20e\%20Detec\%C3\%A7\%20 2005-2014.pdf

4. Cunha MD, Cavaliere FAM, Hércules FM, Duraes SMB, Oliveira MLWDR, Matos HJ. The impact of leprosy elimination strategy on an endemic municipality in Rio de Janeiro State, Brazil. Cad Saude Publica 2007; 23(5):1187-1197.

5. Kerr-Pontes LR, Montenegro AC, Barreto ML, Werneck GL, Feldmeier H. Inequality and leprosy in Northeast Brazil: an ecological study. Int J Epidemiol 2004; 33(2):262-269.

6. Imbiriba ENB, Neto ALS, Souza WV, Pedrosa V, Garnelo L. Social inequality, urban growth and leprosy in Manaus: a spatial approach. Rev Saude Publica 2009; 43(4):656-665.

7. Boerma JT, Weir SS. Integrating demographic and epidemiological approaches to research on HIV/AIDS: the proximate-determinants framework. J Infect Dis 2005; 191(Supl. 1):S61-S67.

8. Adimora AA, Schoenbach VJ. Social context, sexual networks, and racial disparities in rates of sexually transmitted infections. J Infect Dis 2005; 191(Supl. 1):S115-122.

9. Diez-Roux AV. Bringing context back into epidemiology: variables and fallacies in multilevel analysis. $A m \mathrm{~J}$ Public Health 1998; 88(2):216-222.

10. Halloran ME, Struchiner CJ. Causal inference in infectious diseases. Epidemiology 1995; 6(2):142-151. 
11. Cliff AD, Hagget P, Raynor MS. The global sample: an overall picture. In: Cliff A, Haggett P, Smallman-Raynor M, editors. Deciphering global epidemics: analytical approaches to the disease records of world cities. New York: Cambridge University Press; 1998. p. 450.

12. Gálea S, Freudenberg N, Vlahov D. Cities and population health. Soc Sci Med 2005; 60(5):1017-1033.

13. Lapa TM, Albuquerque MFPM, Carvalho MS, Silveira Júnior JC. Análise da demanda de casos de hanseníase aos serviços de saúde através do uso de técnicas de análise espacial. Cad Saude Publica 2006; 22(12):25752583.

14. Opromolla PA, Dalben I, Cardim M. Geostatistical analysis of leprosy cases in the State of São Paulo, 19912002. Rev Saude Publica 2006; 40(5):907-913.

15. Fischer EAJ, Pahan D, Chowdhury SK, Richardus JH. The spatial distribution of leprosy cases during 15 years of a leprosy control program in Bangladesh: An observational study. BMC Infectious Diseases 2008; 8:126.

16. Rodrigues-Júnior AL, Tragante OV, Motti VG. Spatial and temporal study of leprosy in the state of São Paulo (Southeastern Brazil), 2004-2006. Rev Saude Publica 2008; 42(6):1012-1020.

17. Joshua V, Gupte MD, Bhagavandas M. A Bayesian approach to study the space time variation of leprosy in an endemic area of Tamil Nadu, South India. Int $J$ Health Geographics 2008; 7:40.

18. Silva DRX, Ignotti E, Santos RS, Hacon SS. Hanseníase, condições sociais e desmatamento na Amazônia brasileira. Rev Panam Salud Publica 2010; 27(4).

19. Queiroz JW, Dias GH, Nobre ML, Sousa Dias MC, Araújo SF, Barbosa JD, Bezerra da Trindade-Neto P, Blackwell JM, Jeronimo SM. Geographic information systems and applied spatial statistics are efficient tools to study Hansens disease (leprosy) and to determine areas of greater risk of disease. Am J Trop Med Hyg 2010; 82(2):306-314.

20. Penna MLF, Oliveira MLWR, Penna G. Spacial Distribuition of Leprosy in the Amazon Region of Brazil. Emerg Infect Dis 2009; 15(4):650-652.

21. Brook CE, Beauclair R, Ngwenya O, Worden L, Ndeffo-Mbah M, Lietman TM, Satpathy SK, Galvani AP, Porco TC. Spatial heterogeneity in projected leprosy trends in India. Parasit Vectors 2015; 8(1):542.

22. Barreto JG, Bisanzio D, Guimarães LS, Spencer JS, Vazquez-Prokopec GM, Kitron U, Salgado CG. Spatial analysis spotlighting early childhood leprosy transmission in a hyperendemic municipality of the Brazilian Amazon region.PLoS Negl Trop Dis 2014; 8(2):e2665.

23. Duarte-Cunha M, Souza-Santos R, Matos HJ, Oliveira ML. Epidemiological aspects of leprosy: a spatial approach. Cad Saude Publica 2012; 28(6):1143-1155

24. Alencar CH, Ramos Júnior AN, Santos ES, Richter J, Heukelbach J. Clusters of leprosy transmission and of late diagnosis in a highly endemic area in Brazil: focus on different spatial analysis approaches. Trop Med Int Health 2012; 17(4):518-525.
25. Queiroz JW, Dias GH, Nobre ML, De Sousa Dias MC, Araújo SF, Barbosa JD, Bezerra da Trindade-Neto P, Blackwell JM, Jeronimo SM. Geographic information systems and applied spatial statistics are efficient tools to study Hansen's disease (leprosy) and to determine areas of greater risk of disease. Am J Trop Med Hyg 2010; 82(2):306-314.

26. Cabral-Miranda W, Chiaravalloti Neto F, Barrozo LV. Socio-economic and environmental effects influencing the development of leprosyin Bahia, north-eastern Brazil. Trop Med Int Health 2014; 19(12):1504-1514.

27. Freitas LR, Duarte EC, Garcia LP. Leprosy in Brazil and its association with characteristics of municipalities: ecological study, 2009-2011. Trop Med Int Health 2014; 19(10):1216-1225.

28. Sampaio PB, Bertolde AI, Maciel EL, Zandonade E. Correlation between the spatial distribution of leprosy and socioeconomic indicators in the city of Vitória, State of ES, Brazil. Lepr Rev 2013; 84(4):256-265.

29. Cury MR, Paschoal VD, Nardi SM, Chierotti AP, Rodrigues Júnior AL, Chiaravalloti-Neto F. Spatial analysis of leprosy incidence and associated socioeconomic factors. Rev Saude Publica 2012; 46(1):110-118.

30. Cunha MD, Cunha GM, Snatos RS. Geographical heterogeneity in the analysis of factors associated with leprosy in an endemic area of Brazil: are we eliminating the disease? BMC Infect Dis 2015; 15:196.

31. Brasil. Ministério da Saúde (MS). 2002. Guia para controle da Hanseníase. [acessado 2014 dez 10]. Disponível em: http://bvsms.saude.gov.br/bvs/publicacoes/guia_de_hanseniase.pdf

32. Assunção RM, Barreto SM, Guerra HL, Sakurai E. Mapas de taxas epidemiológicas: uma abordagem Bayesiana. Cad Saude Publica 1998; 14(4):713-723.

33. Brasil. Ministério da Saúde (MS). 2009. Hanseníase no Brasil. Dados e Indicadores selecionados. [acessado 2015 out 29]. Disponível em: http://www.morhan.org. br/views/upload/caderno_de_indicadores_hanse_brasil_01_a08_atual.pdf

34. Souza WV, Barcellos CC, Brito AM, Carvalho MS, Cruz OG, Albuquerque MFM, Alves KR, Lapa TM. Aplicação do modelo bayesiano empírico na análise espacial da ocorrência de hanseníase. Rev Saude Publica 2001; 35(5):474-480.

35. Penna ML, Oliveira ML, Penna GO. The epidemiological behaviour of leprosy in Brazil. Lepr Rev 2009; 80(3):332-344.

Artigo apresentado em 11/05/2015

Aprovado em 21/01/2016

Versão final apresentada em 23/01/2016 